\title{
INTERFERON ALPHA INDUCING PROPERTY OF CORONAVIRUS PARTICLES AND PSEUDOPARTICLES
}

\author{
P. Baudoux, ${ }^{1}$ L. Besnardeau, ${ }^{1}$ C. Carrat, ${ }^{1}$ P. Rottier, ${ }^{2}$ B. Charley, ${ }^{1}$ and \\ H. Laude ${ }^{1}$ \\ ${ }^{1}$ Unité de Virologie Immunologie Moléculaires \\ INRA, Jouy-en-Josas, France \\ ${ }^{2}$ Institute of Virology \\ Veterinary Faculty \\ Utrecht University, The Netherlands
}

\section{ABSTRACT}

Previous work in our laboratory have provided evidence that the membrane glycoprotein $\mathrm{M}$ of TGEV is centrally involved in efficient induction of alpha interferon (IFN- $\alpha$ ) synthesis by non-immune peripheral blood mononuclear cells incubated with fixed, TGEV-infected cells or inactivated virions. Here we report recent completion of studies initiated to get a better understanding of the nature of the interferogenic determinant(s). Transfected cells expressing TGEV $\mathrm{M}$ together with the minor structural component $\mathrm{E}$ (formerly called $\mathrm{sM}$ ) were found to trigger IFN- $\alpha$ synthesis. Co-expression of these two proteins was shown to be necessary and sufficient for assembly and release of pseudoparticles resembling TGEV virions. Purified pseudoparticles exhibited an interferogenic activity close to that of authentic virions. Chimeric recombinant particles expressing BCV M ectodomain also induced IFN. Examination of cell cultures infected by viruses representative of the three $\mathrm{Ni}$ dovirales genera revealed that the capacity to act as an efficient IFN- $\alpha$ inducer is a common feature of viral particles of the coronavirus genus. Altogether these data bring new insights regarding the putative nature of the viral structure involved in IFN- $\alpha$ induction.

\section{INTRODUCTION}

The replication of transmissible gastroenteritis virus (TGEV), a highly enteropathogenic virus of swine, primarily takes place in the differenciated enterocytes of the small 
intestine. In infected animals, however, high titers of interferon (IFN) are detected at both the local and systemic levels, and the IFN activity essentially belongs to the alpha type (La Bonnardière and Laude, 1981). Accordingly, the infected enterocytes are unlikely to represent the major IFN- $\alpha$-producing cells. Interfaced investigations involving two groups of our laboratory were being pursued in an attempt to elucidate the nature of the IFN- $\alpha$-producing cells and the mechanism by which TGEV triggers such an efficient synthesis.

An ex vivo induction system was set up in which swine peripheral blood mononuclear cells (PBMC) were used as a source of IFN-producing cells. Incubation of these cells with fixed, TGEV-infected cultures or with UV-inactivated, purified virions results in a strong and early IFN- $\alpha$ synthesis (Charley and Laude, 1988). This led to the conclusion that the IFN- $\alpha$ synthesis is triggered by non-replicating, inert viral structures, a mechanism also shown to be operative for a number of other viruses (for a review, see Fitzgerald-Bocarsly, 1993). The characterization of the IFN- $\alpha$-producing cells is still in progress, but there is now clear evidence that they represent a very minor subpopulation of leukocytes (Riffault et al., 1997 and ref. thererin). The available information concerning the nature of the interferogenic determinants is overall scarce. In the case of TGEV, several lines of evidence support the view that the M protein plays a key role in IFN induction. Firstly, within a collection of antibodies directed to the structural proteins, only two blocked the IFN induction. Both of them recognized the ectodomain of the M protein (Charley and Laude, 1988), which is about 35 amino acid-long after cleavage of the signal peptide (Laude et al., 1987). Secondly, two of the epitope mutants selected towards the above antibodies exhibited a markedly decreased interferogenic activity. Sequencing of the mutant $\mathrm{M}$ genes revealed that the substitutions all mapped within the $\mathrm{N}$-terminal, ectodomain of the protein and impaired its glycosylation without altering the virus infectivity (Laude et al., 1992).

The aim of the present paper is to briefly report our research progress regarding this phenomenon. Two major issues which have been addressed during the past few years are: i) would the single or combined expression of recombinant TGEV M protein induce IFN synthesis ?; ii) is the strong IFN inducer property a unique feature of TGEV or is it shared by other coronaviruses?

\section{MATERIALS AND METHODS}

The procedures for preparation of swine PBMC, induction of IFN by TGEV-infected cultures and IFN- $\alpha$ titration by ELISA have been described earlier (Charley and Laude, 1988; Riffault et al., 1997). Purification of TGEV pseudoparticles and immunoprecipitation of labeled viral proteins were performed as already reported (Laude et al., 1986). The pcDNA1 or pTM1-based plasmid constructs used to express viral proteins under control of $\mathrm{T} 7$ promotor are described in a separate publication (Baudoux et al., submitted). Tetracycline-controlled expression of TGEV genes was done by using the pUHD15.1 and pUHD10.3 plasmid vectors designed by M. Gossen \& H. Bujard (1992).

The origin of viruses and cell lines introduced in the laboratory for IFN induction assays is as follows: FIPV and FEA cells: VIRBAC R \& D Biologie (Le Carros, France); MHV A59 and L cells, EAV and BHK21 cells, BEV and Equine. Dermis cells: Institute of Virology (Utrecht, The Netherlands); IBV and Vero cells, CCV: Rhône-Mérieux (Lyon, France). The origin of the other viruses and cells listed in Table 1 are mentioned in our earlier publications. 
Table 1. Induction of alpha interferon in swine leucocytes incubated with cell monolayers infected by coronaviruses and other members of the Nidovirales

\begin{tabular}{|c|c|c|c|c|c|}
\hline Genus & Virus & Strain & Cell line & Fixation' ${ }^{\prime}$ & IFN- $\alpha$ titre $^{2}$ \\
\hline \multirow{13}{*}{$\begin{array}{c}\text { Coronavirus } \\
\text { (group 1) }\end{array}$} & TGEV & Purdue 115 & ST & - & 17500 \\
\hline & & & & + & 16400 \\
\hline & FIPV & 1146 (type 2) & FEA & - & 4800 \\
\hline & & & & + & 3300 \\
\hline & & & FCWF & - & 1800 \\
\hline & & & & + & 400 \\
\hline & $\mathrm{CCV}$ & IRC5 & FEA & + & 1330 \\
\hline & & & FCWF & - & 550 \\
\hline & & & & + & 510 \\
\hline & $\mathrm{HCV}$ & $229 \mathrm{E}$ & FCWF & - & 5400 \\
\hline & & & & + & 330 \\
\hline & PEDV & CV777 & VERO & - & 680 \\
\hline & & & & + & 680 \\
\hline \multirow{4}{*}{$\begin{array}{c}\text { Coronavirus } \\
\text { (group 2) }\end{array}$} & MHV & A59 & $\mathrm{L}$ & - & 5400 \\
\hline & & & & + & 1100 \\
\hline & $\mathrm{BCV}$ & F15 & HRT 18 & - & 620 \\
\hline & & & & + & 50 \\
\hline \multirow{4}{*}{$\begin{array}{l}\text { Coronavirus } \\
\text { (group 3) }\end{array}$} & IBV & Mass.41 & VERO & - & 11800 \\
\hline & & & & + & 2400 \\
\hline & & Beaud. & & - & 7300 \\
\hline & & & & + & 1500 \\
\hline \multirow[t]{2}{*}{ Torovirus } & BEV & & Eq. Dermis & - & $\leq 10$ \\
\hline & & & & + & $\leq 10$ \\
\hline \multirow[t]{2}{*}{ Arterivirus } & EAV & Bucyrus & BHK21 & - & $\leq 10$ \\
\hline & & & & + & $\leq 10$ \\
\hline
\end{tabular}

${ }^{1}$ The cell sheets were fixed (+) or not $(-)$ with glutaraldehyde.

${ }^{2} \mathrm{IFN}-\alpha$ titre was $\leq 10 \mathrm{IU} / \mathrm{ml}$ in mock-infected cells.

\section{RESULTS}

\subsection{IFN Induction by Cells Co-Expressing TGEV $M$ and Other Viral Proteins}

The vaccinia virus/T7-driven system was used to express $M$ protein in different combinations with the other structural proteins: S (spike), $\mathrm{N}$ (nucleocapsid) and the minor small membrane protein (Godet et al., 1992). For the latter, the designation sM used in previous publications was changed to E according to a recommendation of the ICTV study group made during the Segovia symposium. Fig. 1 shows the IFN- $\alpha$ activity induced by these overexpressing cultures following glutaraldehyde fixation and incubation with PBMC. As a main result, IFN induction occurred only with monolayers co-transfected with the $\mathrm{M}$ and $\mathrm{E}$ genes at least. This finding is consistent with results from our earlier experiments in which stable or transient expression of $\mathrm{M}$ protein in the absence of other viral components failed to induce IFN (Baudoux et al., 1995). The additional expression of S and/or $\mathrm{N}$ did not result in appreciably higher IFN levels. Altogether these data led to the conclusion that the co-expression of $\mathrm{M}$ and $\mathrm{E}$ was necessary and sufficient to generate in- 


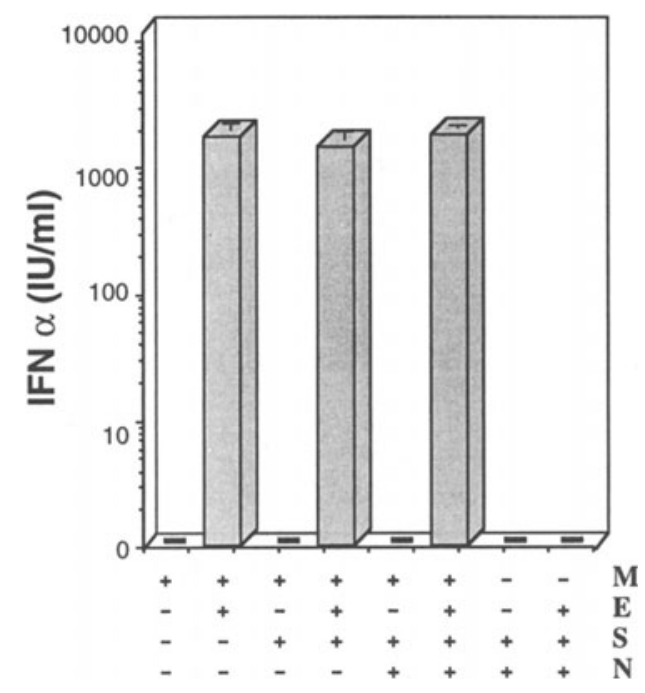

Figure 1. Induction of IFN- $\alpha$ by cell cultures expressing TGEV structural proteins. Vaccinia virus vT7-3-infected RK13 cells were lipofected with one or a combination of plasmids each encoding one of the TGEV genes, as indicated. The monolayers were fixed by $0.25 \%$ glutaraldehyde at $10 \mathrm{hrs}$ p.i. then incubated overnight with PBMC $\left(10^{7} / \mathrm{ml}\right)$. Interferon titer in the maintenance medium was measured by an ELISA assay specific for IFN- $\alpha$.

terferogenic material. The measured IFN titres reached values ${ }^{3} 10^{3} \mathrm{UI} / \mathrm{ml}$, which are close to the titer induced by TGEV-infected, fixed ST cells used for comparison in this assay; in addition, IFN induction appeared to be in correlation with an increased expression of $M$ antigen as detected by an ELISA test using Mabs directed to the M ectodomain (data not shown).

\subsection{TGEV Pseudoparticles Are Released from Cells Co-Expressing the $M$ and $E$ Proteins}

The above data strongly suggested that a direct or indirect interaction with $\mathrm{E}$, a protein shown to be present at a low copy number in TGEV virions (Godet et al., 1992), was absolutely required for the $\mathrm{M}$ protein to acquire an interferogenic structure and/or to gain access to IFN-producing cells. This possibility was reinforced by the recent demonstration by Vennema and coworkers (1996) that co-expression of the MHV M and E proteins led to the assembly and release of particles resembling authentic coronavirions. Experiments were therefore designed to formally assess this point in the case of TGEV. As shown in Fig. 2A, M material was immunoprecipitated from the supernatant of metabolically labelled cell cultures in which $M$ and $E$ were expressed through the VV/T7 vector (right panel), thus demonstrating that soluble $\mathrm{M}$ material was released from cells co-expressing these two proteins. As it can be noted, only a low proportion of the $M$ material synthesized intracellularly was secreted in this experiment, and the level of E protein expression appeared to be a limiting factor as the amount of extracellular $\mathrm{M}$ increased with the concentration of E-encoding plasmid used for transfection. In subsequent experiments the assay was improved but the proportion of released material never exceeded $10 \%$ of the synthesized M. This may indicate that the assembly and/or release in this model system is less efficient than in infected cells, where other viral components such as non-structural polypeptides could play a role so as to enhance this process. Investigations are currently being performed in order to address this point. 
A

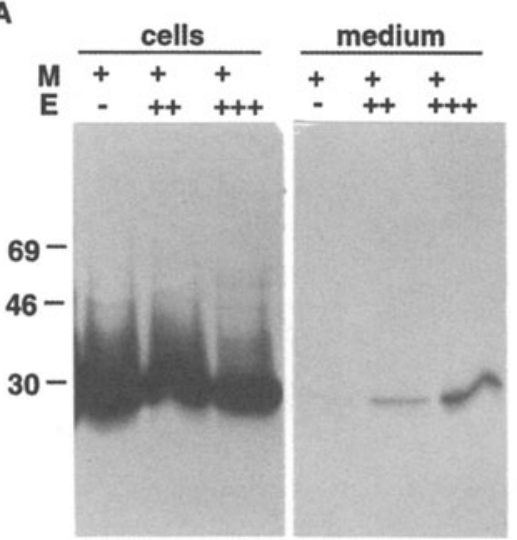

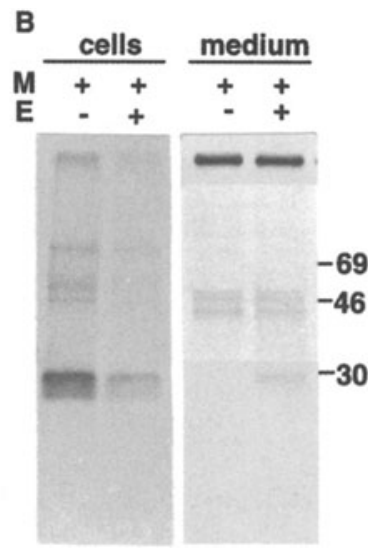

Figure 2. Secretion of TGEV M protein through co-expression of $M$ and $E$ gene constructs. (A) vT7-3-infected monolayers were transfected with pcsM (E) and pTM2 (M) plasmids as indicated, and labelled with ${ }^{35}$ S $]$ methionine. Viral proteins from the cell lysates and culture media were immunoprecipitated by anti-M monoclonal antibodies and analyzed by SDS-PAGE electrophoresis. (B) Cells were transfected with constructs allowing a tetracycline controlled expression of $\mathrm{M}$ and $\mathrm{E}$ genes. The resulting material was processed as above. The medium lanes are shown after a 10 -fold prolonged exposure.

To rule out the possibility that release of $M$ protein occurred merely as a consequence of the infection by vaccinia virus, an additional experiment was performed in which TGEV $M$ and $E$ proteins were expressed through an alternative system based on the use of tetracycline-dependent plasmid expression vectors. As shown in Fig. 2B, release of $M$ protein occurred as well, thus supporting the view that it was a relevant phenomenon rather that an artifact resulting from the use of the VV/T7 vector. Finally, ultracentrifugation in sucrose gradient revealed that isotopically labelled $M$ material released from transfected, VV/T7-infected cells sedimented as a discrete peak, at a slightly slower rate than TGEV virions (not shown). Overall these data led us to conclude that TGEV E and M proteins interactions allow the formation of pseudoparticles, similar to that reported for MHV.

\subsection{Purified TGEV Pseudoparticles Are Potent IFN- $\alpha$ Inducers}

A key issue was whether the specific interferogenic activity of the so obtained recombinant particles compared that of TGEV virions. To address it, a stock of pseudoparticles produced in transfected, VV/T7 infected RK13 cells was prepared and purified following the same procedure as used for TGEV virions. When examined in electron microscopy, the resulting preparation was shown to contain particles which looked like authentic virions except that they were devoid of prominent, typical coronavirus spikes (Fig. 3). It was also noted that, consistent with the absence of a nucleocapsid core, the pseudoparticles had a tendency to collapse, which implies a real flexibility of the coronavirus envelope.

Suspensions of purified pseudoparticles and virions having the same $M$ antigen content were allowed to incubate with PBMC. The IFN titers induced by the two types of material were found to be of the same order of magnitude, thus establishing that pseudoparticles 


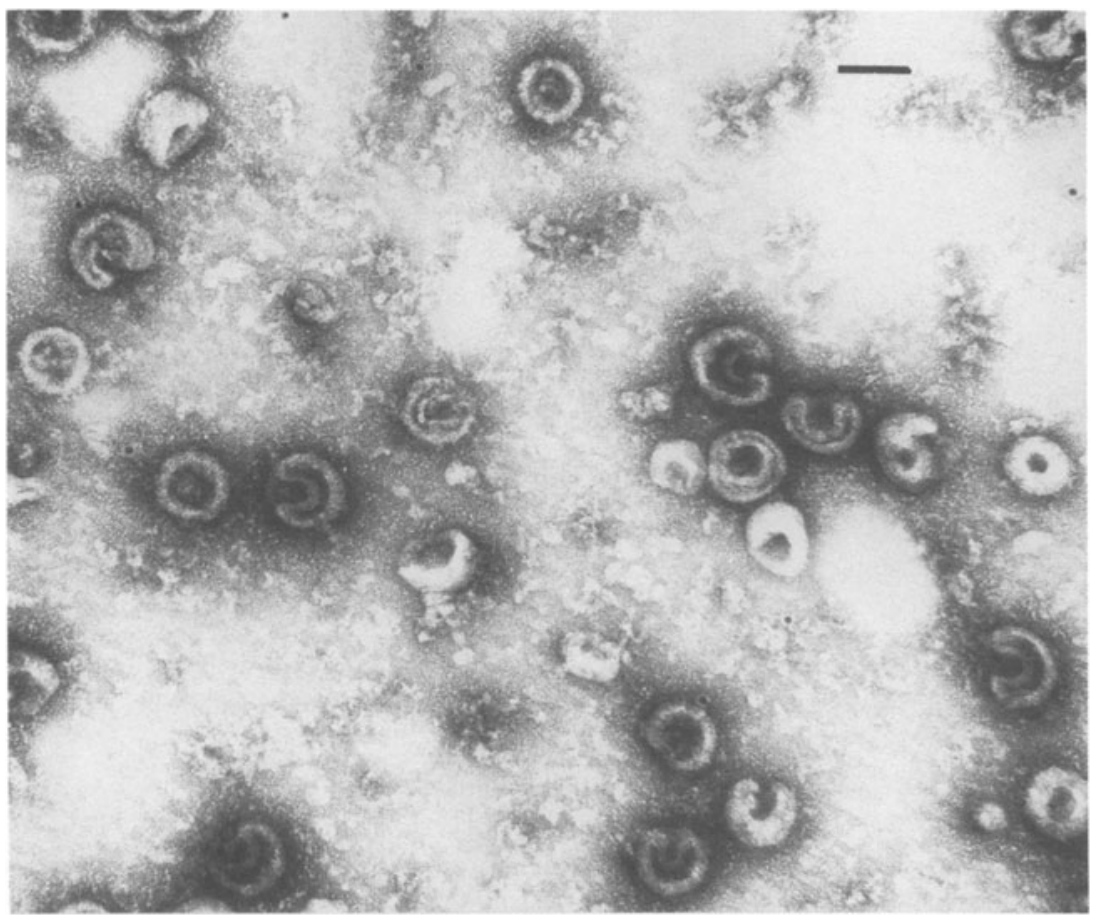

Figure 3. Electron microscopy of TGEV pseudoparticles. Particles released from RK 13 cells expressing M and E genes through the VV/T7 vector were purified in a sucrose gradient. Particles were viewed after negative staining (bar: 100nm).

were nearly as potent IFN inducer as TGEV virions (Fig. 4). Moreover, the addition of appropriate anti-M ectodomain monoclonal antibodies during the incubation strongly inhibited the IFN induction by pseudoparticles, similar to that already observed with TGEV. This confirmed that identical determinants were involved in both cases.

\subsection{IFN Induction by a TGEV/BCV Chimeric M Protein}

The possibility of generating fully interferogenic, TGEV-like particles from engineered genes opened the way to closer investigations regarding the nature of the molecular determinants responsible for such an activity. As a first approach, it was decided to generate TGEV/BCV chimeric particles by taking advantage of BCV $\mathrm{M}$ genes previously cloned in the laboratory (Savoysky et al., 1990; Woloszyn et al., 1990). As will be described in a separate paper (Baudoux et al., submitted), a general outcome of these experiments was that the co-expression experiments using constructs encoding chimeric either $\mathrm{M}$ or $\mathrm{E}$, or both, did not allow particle formation at a workable level. A noticeable exception was the M chimera called BTT, in which the BCV ectodomain was swapped in the TGEV M sequence. Not only the BTT chimera lead to relatively efficient particle forma- 
Figure 4. IFN- $\alpha$ inducing activity of pseudoparticles and virions of TGEV. Purified preparations of particles were adjusted to have the same content in $\mathrm{M}$ antigen as judged by ELISA assay, and incubated with PBMC at the indicated dilutions. The blocking effect of anti-M monoclonal antibodies on IFN induction is shown on the right.

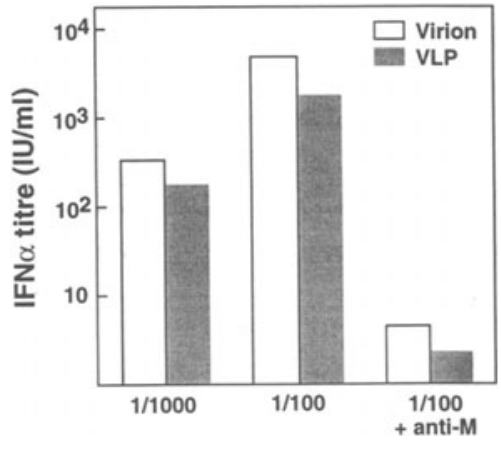

tion, but also it exhibited a limited specificity of interaction with $\mathrm{E}$ (data not shown). When analyzed for IFN induction, BTT was found to promote IFN synthesis as efficiently as homologous TGEV M when coexpressed with TGEV E (Fig. 5). Co-expression of BTT with BCV E also induced IFN, although at an appreciably lower level, which was correlated to a less efficient particle formation. Last but not least, cultures co-expressing the homologous BCV $\mathrm{M}+\mathrm{E}$ pair exhibited also substantial interferogenic activity.

\subsection{Replication-Independent Induction of IFN- $\alpha$ Is a Common Feature of Coronaviruses}

The finding that, like TGEV, BCV pseudoparticles were interferogenic raised the question of whether this property could be shared by other coronaviruses or by other members of the Nidovirales. To answer it, cultures infected by one each of the ten different viruses listed in Table 1 were produced using appropriate permissive cell systems. After onset of the cytopathic effect, fixed or unfixed monolayers were allowed to incubate with swine leukocytes as a source of IFN-producing cell, and the IFN-a titre associated with the resulting supernatants was determined by ELISA assay. As a striking result, all the seven coronaviruses tested, which are representative from the three genetic subsets of the genus, were able to trigger a substantial IFN synthesis, like TGEV. Importantly, IFN

Figure 5. Induction of IFN- $\alpha$ by cell cultures expressing homologous or chimeric pseudoparticles of TGEV and BCV. The experiment was performed as in Fig. 1. The expressed viral genes are designated as uppercase letters on abscissa axis for $\mathrm{M}$ constructs, and lowercase letters in insert for E constructs. T and $t$ for TGEV, $\mathrm{B}$ and $\mathrm{b}$ for $\mathrm{BCV}$. BTT designates a chimeric $\mathrm{M}$ gene (see text).

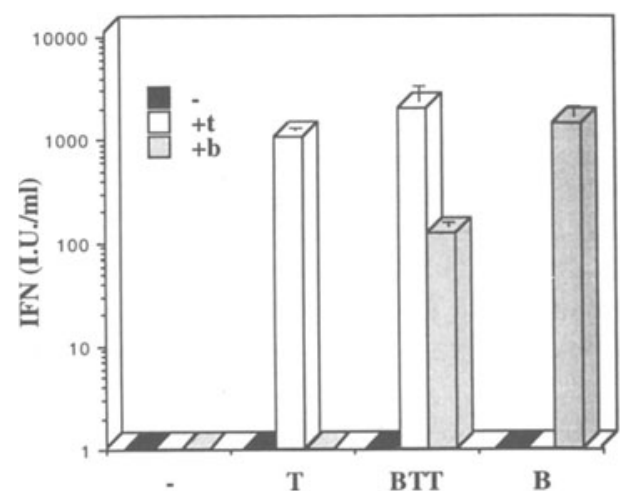


production persisted after glutaraldehyde fixation of the monolayers prior to incubation with the leukocytes. For several infected cultures, the IFN titer decreased after fixation, possibly due to the fact that synthesis of interferogenic material was blocked by fixation while it proceeded during incubation with unfixed cultures. An inconstant effect of fixation on the IFN titer has been recognized in the course of our experiments with TGEV. Alternatively, the integrity of the interferogenic determinants may be affected by glutaraldehyde to variable extents differing with the virus tested. In any case, these data demonstrate that the property of triggering IFN synthesis in leukocytes by recognition of inert viral structures is shared by many coronaviruses. In contrast, Berne virus (BEV) and equine arteritis virus (EAV), representative of the two other nidovirus genera, failed to induce IFN in two separate assays in the same above conditions. In an independent study, the porcine reproductive respiratory syndrome virus (PRRSV, arterivirus) failed to induce an in vitro IFN- $\alpha$ response in swine PBMC (E. Albina; personnal communication). It remains possible, however, that latter viruses would act as inducers for leukocytes from other species than the swine.

The experiments using infected cultures as inducers do not allow to draw valid conclusions regarding the respective interferogenic activity of the different coronaviruses since the amount of viral material produced may vary significantly among the infected cultures and was not quantified. Moreover, PBMC from different batches were used for induction. The only way to address this point is to use suspensions of purified virions at identical concentrations as inducers in the same assay, which was done for two viruses in addition to TGEV. $\beta$-propiolactone-inactivated FIPV virions incubated with swine PBMC $\left(1 \mu \mathrm{g} / 2 \times 10^{6}\right.$ cells) induced IFN titers of $4-5 \times 10^{3} \mathrm{IU} / \mathrm{ml}$, close to what UV-inactivated TGEV virions induced in the same assay $\left(7-8 \times 10^{3} \mathrm{IU} / \mathrm{ml}\right)$. BCV, however, appeared to be a less potent inducer than the two above viruses, with IFN titres of 3-4 $310^{2} \mathrm{UI} / \mathrm{ml}$, a result which can be correlated to the relatively low interferogenic activity of BCV-infected, fixed cultures. This last result was not expected since cultures producing BCV and TGEV recombinant pseudoparticles induced comparable IFN titers. Although this finding would require further examination, one can speculate that the presence on $\mathrm{BCV}$ virions of a second layer of short spikes (HE protein) may result in a partial masking of interferogenic determinants.

\section{DISCUSSION}

The major achievement of these studies was the demonstration that recombinant TGEV-like particles, lacking spikes, nucleocapsid and genomic RNA, may substitute to authentic virions for triggering an efficient IFN- $\alpha$ synthesis by swine leukocytes. The pseudoparticles were generated through the expression of the major virion component, the polytopic transmembrane glycoprotein $\mathrm{M}$, which was previously proposed by us to play a key role as IFN inducer (see introduction), together with the minor, small transmembrane protein $\mathrm{E}$ (formerly called $\mathrm{sM}$ ). These results nicely confirmed the observations recently reported on MHV by Vennema and coworkers and further support the view that, through an interaction whose precise nature remains to elucidate, the $\mathrm{M}$ and $\mathrm{E}$ proteins represent both necessary and sufficient partners for coronavirus assembly. The characteristics of the induction of IFN- $\alpha$ by TGEV pseudoparticles closely resembled those previously established for virions: i) their intrinsic interferogenic activity nearly approached that of TGEV virions, as estimated on the basis of their respective $M$ antigen content; it should be noted in this respect that, for practical reasons, the two types of particles were produced in dis- 
tinct cell systems, and thus may differ to some extent in terms of carbohydrate and lipid composition; ii) antibodies directed to the M ectodomain were able to prevent the IFN induction; iii) co-expression of S spike protein had no discernible effect on the interferogenic activity, in agreement with our earlier finding that anti-S or anti-APN receptor antibodies did not affect IFN stimulation.

Another relevant result of our work was that the ability to act as a potent IFN- $\alpha$ inducer for PBMC is not restricted to TGEV but appears to be a common, so far unrecognized trait of coronaviruses. Indeed, cultures infected by any of the members of the genus tested so far, as well as FIPV and BCV purified virus suspensions and BCV pseudoparticles-producing cells, were found to induce an efficient IFN synthesis by swine leukocytes. There is circumstantial evidence that the strong IFN response observed in TGEV-infected animals is raised through this virus RNA-independent induction pathway. Hence, it might be worth investigating the interferogenic activity of other viruses of the genus towards leukocytes from their respective, natural host species, in order to evaluate any possible biological relevancy of this phenomenon in coronavirus infections.

The mechanism by which coronavirus particles, either free or associated with the cell surface, commit the IFN-producing cells to express the related genes is far from being understood. However, our findings altogether brought truly new insights in this respect. The early step of the induction presumably involves the recognition of a surface-exposed, viral structure. Both theoretical and experimental reasons led us to propose the ectodomain of the TGEV M protein, as a candidate for this function. It appeared, however, that engineered TGEV chimeric pseudoparticles carrying the BCV M ectodomain, which differs from its TGEV counterpart in terms of size, amino acid sequence, and glycosylation type, were as interferogenic as non-chimeric particles. Furthermore, as outlined above, this property is potentially a general feature of the coronavirus particle. Inspection of the amino acid sequences failed to reveal any candidate motif at least in the assumedly exposed regions of the protein. Thus an attractive view emerging from these studies is that the induction could be mediated by a tridimensionnal, possibly repetitive, structure rather than by a sequence motif. Such a hypothetical IFN-inducing determinant should be unique to the coronavirus particle since other Nidoviruses do not appear to share this intriguing biological property.

\section{ACKNOWLEDGMENTS}

We express our deep thanks to Joëlle Cronier and Véronique Duquesne (Virbac) for providing virus, cells and FIPV-inactivated suspension for purification; Gert-Jan Godeke, Twan de Vries and Lisette Cornelissen from Peter Rottier's lab for kindly making available to us MHV, EAV and BEV viruses, and L, BHK21 and Equine Dermis cell lines; Jean-François Bouquet and Gilles Chappuis (Rhône-Mérieux) for IBV and CCV virus strains; Pascal Boireau for providing the cloned $\mathrm{M}$ and $\mathrm{E}$ genes.

\section{REFERENCES}

Baudoux P., Charley B., Laude H., 1995, Recombinant expression of the TGEV membrane protein, in "Coronaand related viruses" (P. Talbot \& G. Levy Eds.), Adv. Exp. Med. Biol. 380: 305-310.

Charley B., Laude H., 1988, Induction of interferon alpha by transmissible gastroenteritis coronavirus : role of transmembrane glycoprotein E1, J. Virol. 62: 8-11.

Fitzgerald-Bocarsly P., 1993, Human Natural Interferon-a producing cells, Pharmacol. Ther. 60: 39-62. 
Godet M., L'Haridon R., Vautherot J.F., Laude H., 1992, TGEV coronavirus ORF4 encodes a membrane protein that is incorporated into virions, Virology 188: 666-675.

La Bonnardière C., Laude H., 1981, High interferon titer in newborn pig intestine during experimentally induced viral enteritis, Infec. Immun. 32: 28-31.

Laude H., Chapsal J.M., Gelfi J., Labiau S., Grosclaude J., 1986, Antigenic structure of transmissible gastroenteritis coronavirus I. Properties of monoclonal antibodies directed against virion proteins, J. Gen. Virol. 67: $119-130$.

Laude H., Gelfi J., Lavenant L., Charley B., 1992, Single amino acid changes in the viral glycoprotein M affect induction of alpha interferon by coronavirus TGEV, J. Virol. 66: 743-749.

Laude H., Rasschaert D., Huet J.C., 1987, Sequence and N-terminal processing of the transmembrane protein El of the coronavirus transmissible gastroenteritis Virus, J. Gen. Virol. 68: 1687-1693.

Riffault S. Carrat C. Besnardeau L., La Bonnardiere C., Charley B., 1997, In vivo induction of interferon-a in pig by non-infectious coronavirus: tissue localization and in situ phenotypic characterization of interferon-a producing cells, J. Gen. Virol. 78: 2483-2487.

Savoysky E., Boireau P., Finance C., Laporte, J.,1990, Sequence and analysis of BECV F15 matrix protein, Res. Virol. 141: 411-425.

Vennema H., Godeke G-J., Rossen J.W.A., Vorhout W.F., Horzinek M.C., Opstelten D.-J., Rottier P.J.M., 1996, Nucleocapsid-independent assembly of coronavirus -like particles by co-expression of viral envelope protein genes, EMBO J. 15: 2020-2028.

Woloszyn N., Boireau P., Laporte J., 1990, Nucleotide sequence of the bovine enteric coronavirus F15 mRNA 5 and mRNA 6 unique regions, Nucl. Ac. Res. 18: 1303. 